\title{
The effects on eye movements of complexity, novelty, and affective tone'
}

TERRY T. FAW AND JUM C. NUNHALLY

VANDERBILT UNIVERSITY

The major purpose of this study was to investigate the effects of stimulus complexity, novelty, and affective tone on the direction of eye movements of male college students. Motion pictures were taken of S's eye while he viewed pairs of stimuli. In no instance, in any part of the $10 \mathrm{sec}$. viewing interval, did Ss as a group fixate longer on unpleasant stimuli when they were paired with either pleasant or neutral stimuli; and pleasant stimuli consistently dominated neutral stimuli. Also, novel stimuli and complex stimuli tended to dominate their non-novel and less complex competitors. Differences in instructions were found to markedly affect the magnitude but not the direction of fixation-dominance.

For many years the word "attention"' had a mentalistic connotation that was not in keeping with the spirit of early behaviorism. In recent years, however, there has been a definite re-emergence of the construct of attention as an important aspect of behavior theory. But, in spite of the obvious importance of attentional processes, very little research has been undertaken to investigate the stimulus characteristics and motivational variables affecting attention. Rather, the emphasis has been on the employment of attention as a construct to account for hypothetical covert processes, e.g., as was done by Mackintosh (1965) with respect to discrimination learning.

The purpose of the present experiment was to lay some foundation stones with respect to the direct measurement of stimulus effects on one aspect of attention-that of fixation-dominance in gross eye movements. As the term will be used here, fixationdominance (FD) is the tendency for Ss to spend more time fixating on one part of a particular visual display rather than on other parts.

There is only a modest number of reported studies either directly or indirectly concerning effects of stimulus variables on FD. Investigators have employed visual fixations as a dependent measure in studying the effects of positive and negative stimuli on approach and avoidance behavior (Webb, Matheny, \& Larson, 1963; Luborsky, Blinder, \& Mackworth, 1963). Others have attempted to condition the direction of eye fixations by reinforcing certain fixation patterns (Solley \& Santoes, reported in Solley \& Murphy, 1960, p. 190). The results from these studies suggest that FD is greater for stimuli with positive affective tone than for stimuli with negative affective tone.

More research has been devoted to the effects of structural variables on FD than has been devoted to the effects of affective tone. The research reported and reviewed suggests that increased FD is associated with increases in novelty and complexity (Berlyne, 1958; Cantor, 1963; Thomas, 1965; Fantz, 1965)。

The research to be reported grew out of attempts to use eye movements as a dependent measure in studying changes in the FD of neutral stimuli (nonsense syllables or geometric forms) as a result of associating those stimuli with positive or negative events (winning or losing pennies, candy, etc.), e.g., Nunnally, Knott, and Duchnowski (in press); Nunnally, Stevens, and Hall (1965). In the experiments conducted thus far, results from non-perceptual measures (e.g., verbal evaluation) have indicated that positive and negative associations were formed. The results from the attentional measure (FD), however, have been inconsistent. This lack of consistent results prompted an examination of the underlying assumption that affective tone is associated with FD.

In the present study, hypotheses are based on the premise that gross eye movements are mainly determined by two variables: affective tone and information value. Here information value refers to the difficulty with which a subject assimilates the content of a visual display. Complexity and novelty are dimensions of this variable. Affective tone refers to the ratings made of stimuli along a continuum of pleasantnessunpleasantness. If the information values of two stimuli are equated, it is hypothesized that Ss will look more frequently at the more positive stimulus. Contrariwise, if affective tone is equated, they will look more frequently at the stimulus with greater information value.

\section{Subjects}

\section{METHOD}

Ss were 48 male undergraduates enrolled in introductory psychology classes at Vanderbilt University. They were randomly assigned to one of three instructional treatment groups.

\section{Apparafus}

The $\mathrm{S}$ sat in front of a viewing box used to present a series of $35 \mathrm{~mm}$ slides. The box was $24 \mathrm{in}$. long and 21 in. high. At one end was a $21 \times 14$ in. screen. The sides of the box tapered in from the screen for 10-1/2 in. until the width of the box was $14 \mathrm{in.}$. At that point, the sides ran parallel to one another for the remaining $13-1 / 2$ in. The box was adjusted so that the $S$ could rest his face in a goggle-like opening in the front and view the white plastic screen at the 
far end. This opening was in line with the midpoint of the screen. A chin rest was used to reduce the $S^{t} \mathrm{~g}$ head movements. Stimulus slides were projected onto the screen from behind the viewing box by a Bell and Howell slide projector. The projector was connected to a timing device which controlled the length of presentation of each slide.

A Bolex $16 \mathrm{~mm}$ movie camera with a $100 \mathrm{~mm}$ lens was mounted on the side of the box. The lens extended through an opening in the box and was focused on a small mirror mounted on the inside. The mirror reflected the image of the S's left eye onto the lens. The eye was photographed using Kodak High Speed Infrared film running at two frames per sec. Except for the viewing screen and a 40-W red light located in the bottom of the box, the experimental room was dark.

\section{Stimuli}

Stimuli consisted of pairs of words or objects photographed on $35 \mathrm{~mm}$ slides and projected onto a viewing screen. The inside edges of the two words or objects were separated horizontally by $10 \mathrm{in}$. or more. The projected image of the slide measured 21 in. wide and $14 \mathrm{in}$. high. The distance between the midpoints of the two stimuli formed an arc of $36020^{\circ}$ as measured from the center of the goggle opening. This forced the $S$ to make a gross movement of his eyes in order to bring either stimulus into his direct line of sight.

Various types of stimulus pairs were employed to increase the generality of the results. They may be divided into two broad categories, concerning either structural variations or variations in affective tone.

Structure. Complexity and novelty were selected as the structural variables for investigation in the present experiment.

(a) Complexity-Stimuli consisted of two randomiygenerated geometric figures selected from each of three levels of complexity as defined by Attneave (1957). The three levels of complexity contained figures having 24,12 , and 4 sides, respectively. The six figures were equated for association value according to the report of Vanderplas and Garvin (1959).

(b) Novelty-From Berlyne's study of the orienting response (1958), two stimulus pairs were taken in which the novel stimulus is described by Berlyne as having incongruent juxtaposition, e.g., a picture of a rabbit paired with a picture of a rabbit whose body had been replaced by that of an automoblle. Two similar pairs were designed by the investigator.

Affective Tone. The investigation of the effect of variations in affective tone on FD involved the comparison of stimuli representing three points along a continuum of affective tone: positive ( + ); neutral (0); and negative $(-)$. Prior to the experiment, a group of 10 judges rated the affective tone of all stimuli except those in the sets labeled money and nudes. The ratings were made on a nine-point scale extending from "extremely pleasant" to "extremely unpleasant." After the experiment, Ss made a similar rating of those stimuli. These ratings indicate that the stimuli within sets were ordered correctly from pleasant to unpleasant.

Pairs of stimuli were drawn from the following classes for use in all three of the possible comparisons between the three levels of affective tone. Except for those stimuli in Class (c) all pictures were achromatic.

(a) Words-Four sets of three words, in which one word had positive, one negative, and one neutral connotation. Words within sets were equated for length, familiarity (Thorndike \& Lorge, 1963), and part of speech.

(b) Facial Expressions-Four sets of pictures showing varied facial expressions. Each set consisted of the same model in three poses-pleasant, unpleasant, and neutral facial expressions. Two of the models were men and two were women.

(c) Colored Photographs of Faces-Four sets of photographs in which one picture was the face of an attractive model, one the face of a person with average facial features, and one a face that had been extremely marred by some physical defect.

In addition to those three classes of stimull, the + vs. 0 comparison included pairs from the following clagses.

(d) Girls-Two pictures of the faces of very attractive girls and two pictures of the faces of girls of average attractiveness.

(e) Clothes-A male model dressed in sport coat and slacks $\mathrm{Vs}$. the same model dressed in dirty work clothes.

(f) Automobiles-Two sets of pictures in which the comparison was a picture of a new and attractive automobile vs, an old, delapidated automobile.

(g) Nudes-Two sets comparing a picture of a female nude with a rectangular display of single digit numbers located on the four corners and center of the rectangle.

(h) Money-The final class of stimuli varied symbolic value, a variable believed to be related to affective tone. The comparison objects were newlyprinted 1, 5, and 20 dollar bills.

All possible combinations of pairs within each set were photographed as stimulus slides. This resulted in two sets of 64 slides each. The sets were identical except that the left-right positions of the pictures on each slide were reversed.

\section{Procedure}

Each $S$ was individually escorted into the experimental room and familiarized with the viewing box. All Ss were told that they would be shown a series of pictures on the screen in the front of the box. In 
addition the Ss in the preference-set group (PS) and disguised-set group (DS) were told that the pictures would appear in pairs. To the Ss in the PS group, $E$ said, "When these pairs of pictures appear, I want you to look at whichever picture you prefer." Here the word "prefer" was emphasized to imply that E was interested in the S's preference. These Ss were told nothing more about the experiment. Ss in the DS group were told that the experiment involved a study of pupil dilation. $E$ said to $S$, "I am interested in examining how the pupil dilates and constricts as a person's eyes are moving. When I show you these pairs of pictures on the screen, it does not matter what you look at, but you must keep your eyes on the screen so so that I can record the size of your pupil. The pictures will simply give you something to look at during the experiment." A third group, no-set (NS), was told only to look at the screen but was told nothing about the nature of the stimulus slides or the purpose of the experiment. These instructional groups were not included to see if instructions can influence FD, for that surely can be done with extremely specific instructions as to what the $S$ is to look at, but, rather, the purpose was to see if alternative instructions which might actually be employed in such research would lead to different results.

After the instructions were given, $\mathrm{S}$ was presented the series of 64 stimulus slides. Each slide was presented for $10 \mathrm{sec}$. and was preceded for $2 \mathrm{sec}$. by a fixation slide consisting of a single asterisk located in the center of the screen. The fixation slides were used to bring the S's line of vision back to the center of the screen before each test slide appeared. All Ss were informed of the fixation slides and were instructed to look directly at the asterisk when it appearea.

To rule out bias due to position preference, the left-right positions of all stimuli were reversed for half the Ss in each treatment group. One of six random orders of presentation was randomly assigned to each $\mathrm{S}$. The presentation was broken into six equal-length intervals, lasting $2.4 \mathrm{~min}$. each. Between the presentation periods, $\mathrm{S}$ was given a rest period of approximately $45 \mathrm{sec}$.

To obtain information about awareness of $\mathrm{FD}, \mathrm{S}$ was reshown the entire series of slides and asked to report which of the two stimuli in each pair he felt he had viewed the longer when that pair of pictures had appeared previously.

Finally, $\mathrm{S}$ was asked to rate each stimulus on a nine-point scale of affective tone. This scale extended from "extremely pleasant" to "extremely unpleasant."

\section{Analysis of Photography}

\section{RESULTS}

The individual movie frames taken of each S's left eye were analyzed by an experienced judge who was uninformed about the stimuli shown to the Ss. Using the corneal reflection as an index of the eye's position, the judge recorded the position of the eye as either right, left, or middle. A second judge made the same analysis on a sample of the film. The interjudge agreement was $97.5 \%$. The score for an individual $S$ was the percentage of time he spent viewing the more pleasant, complex, or novel stimulus in each pair. Center fixations were not used in the analysis.

\section{Overall Results}

An initial examination of the data suggested that there was a period of from 2 to $3 \mathrm{sec}$. at the beginning of each slide when Ss made alternating scanning movements toward the stimuli. Following this initial scanning period, the percentage of time spent viewing the dominant stimulus increased. No subsequent decrease in the percentage of looking toward that stimulus was noted during the remainder of the $10 \mathrm{sec}$. period. Analysis of the data both with and without the inclusion of the first $2 \mathrm{sec}$. of viewing time resulted in the same pattern of significant results. The elimination of the first $2 \mathrm{sec}$, however, was felt to give a more adequate expression of FD; therefore, all analyses reported were made using percentages based on that data.

\section{Insfructions}

Because of the various sets of stimuli employed in the experiment, six separate analyses of variance were performed to investigate the effects of instructions on subsequent FD. Table 1 summarizes the means and standard deviations of FD for the three instructional groups on the six types of comparisons that were used in those analyses of variance. Except for those sets of comparisons labeled "money" and "complexity," the analyses were based on the mean of the S's separate FD scores for the subsets of stimuli appropriate to the particular comparison, e.g., the mean FD of all subsets comparing $(+)$ and $(-)$ affective tone. The two exceptions were based on the means of the S's FD of all stimulus comparisons within the appropriate class, e.g., all the possible

\section{Table 1.}

Mean Percentage of Viewing Time (FD) for the More Positive, Complex or Novel Stimulus-All Relevant Stimulus Sets Combined

\begin{tabular}{lccccccc} 
& \multicolumn{2}{c}{ PS } & \multicolumn{3}{c}{ Instructions } & \multicolumn{2}{c}{ DS } \\
& & $M$ & SD & $M$ & SD & $M$ & SD \\
\hline \multirow{3}{*}{ Affect } & $+/ 0$ & 83.2 & 09.4 & 64.4 & 09.5 & 59.1 & 06.9 \\
& $0 /-$ & 74.7 & 17.0 & 55.1 & 11.9 & 55.7 & 10.0 \\
Money & $+/-$ & 81.6 & 17.7 & 59.9 & 13.1 & 55.2 & 11.8 \\
Complexity & & 85.3 & 18.5 & 61.8 & 18.4 & 58.2 & 12.2 \\
Novelty & 55.0 & 26.6 & 60.5 & 07.8 & 59.1 & 09.1 \\
& & 35.0 & 25.4 & 53.7 & 08.7 & 58.6 & 14.2 \\
\hline
\end{tabular}


comparisons of three levels of complexity or monetary value. These last two sets of means do not represent FD when two specific levels of monetary value or complexity are compared; rather, they are general indices of the overall FD of more complex or valued objects when compared to less complex or valued objects.

With the exception of the analysis based on comparisons of levels of complexty, all analyses showed significant differences among instructional groups beyond the .01 level. The Neuman-Keuls test of multiple comparisons showed that for all five analyses there were differences $(p<.01)$ between the PS group and the two other groups. There were no statistically significant differences between the NS and DS groups. The analysis involving a comparison of more and less complex figures yielded a nonsignificant $F$ ratio.

\section{Stimulus Variations}

Affective Tone and Money. In all four comparisons shown in Table 1 involving variations of affective tone and money there is a tendency for the more positive or valuable stimuli to dominate attention. This is most evident in the PS group where by $t$ test all such comparisons result in significantly more attention being directed towards the more positive or valued stimuli $(p<.01)$. The other two groups also show this tendency, although to a lesser extent. Previous analyses of variance of the four comparisons showed no signiflcant differences between the means of the NS and DS groups; consequently, the results from these two groups were combined to determine if the FD differed from the chance level of .50 . In all four comparisons the probability of FD being equal to or less than .50 was by $t$ test less than .01 .

Table 2 summarizes the means and standard deviations of FD for the three instructional groups for all comparisons within each of the various sets of stimuli. Examination of Individual sets of stimuli included in the six overall comparisons shows the same trends as those mentioned with regard to the combined data presented in Table 1 . It is striking to see that, of the 48 comparisons of levels of affective tone or money, 46 conform to the hypothesis that greater positive affective tone or value is associated with greater FD. Another important point to note is that in no instance, in any group, in any part of the viewing time, did a negative or less valuable stimulus significantly dominate either a positive, neutral, or more valuable stimulus.

Complexity. An analysis of variance showed no differences between the instructional groups in their FD for more complex stimuli. The FD of more complex stimuli for the combined groups was significantly greater than the chance level of $.50, t=3.280$, df $=$ $47, p<.01$. If one looks at the individual comparisons among the three levels of complexty presented in Table 2, it appears that in all cases the PS in-
Table 2. Mean Percentage of Viewing Time (FD) for the More Positive or Complex Stimulus

\begin{tabular}{lccccccc} 
& & \multicolumn{9}{c}{ Instructions } \\
& & $M$ & SD & $M$ & SD & $M$ & SD \\
\hline \multirow{3}{*}{ Words } & $+/ 0$ & 77.3 & 16.6 & 56.8 & 11.1 & 55.2 & 12.4 \\
& $0 /-$ & 67.1 & 25.9 & 53.9 & 14.2 & 59.0 & 15.3 \\
Facial & $+/-$ & 74.5 & 27.4 & 57.0 & 17.2 & 55.5 & 20.7 \\
Expressions & $+/ 0$ & 83.3 & 15.1 & 61.7 & 13.0 & 55.0 & 11.3 \\
& $0 /-$ & 80.8 & 19.0 & 59.2 & 14.8 & 56.8 & 15.0 \\
Colored & $+/-$ & 87.8 & 18.7 & 61.6 & 15.1 & 54.7 & 14.2 \\
Pictures & $+/ 0$ & 86.2 & 10.7 & 69.7 & 11.7 & 65.0 & 15.8 \\
of Foces & $0 /-$ & 76.1 & 20.6 & 46.0 & 19.4 & 51.4 & 16.8 \\
Girls & $+/-$ & 82.6 & 23.0 & 61.1 & 19.1 & 55.2 & 18.2 \\
Automobiles & $+/ 0$ & 89.6 & 15.5 & 67.2 & 13.2 & 61.4 & 13.8 \\
Clothes & $+/ 0$ & 85.8 & 25.8 & 64.2 & 12.3 & 57.7 & 12.1 \\
Nudes & $+/ 0$ & 64.7 & 38.0 & 61.9 & 19.9 & $48.4 * * 14.9$ \\
& $+/ 0$ & 95.4 & 08.6 & 69.6 & 20.7 & 70.1 & 14.0 \\
Money & $\$ 20 / 5$ & 85.4 & 29.6 & 63.8 & 24.0 & 62.7 & 18.0 \\
& $\$ 5 / 1$ & 80.9 & 30.5 & 56.0 & 24.6 & $53.3 *$ & 23.9 \\
& $\$ 20 / 1$ & 89.6 & 25.2 & 59.2 & 26.1 & 59.5 & 16.1 \\
Complexity & $24 / 12$ & 46.0 & 33.4 & 52.1 & 16.4 & 52.8 & 13.5 \\
& $12 / 4$ & 59.6 & 33.6 & 60.3 & 09.4 & 56.4 & 11.2 \\
& $24 / 4$ & 59.5 & 34.1 & 67.9 & 11.0 & 68.1 & 14.9 \\
\hline
\end{tabular}

$N=15$

$* * N=14$

structions deflated the FD for more complex stimuli. Only in the NS and DS groups do these individual comparisons result in FDs that were significantly different from the chance level. The subcomparisons of the most complex stimuli (24 sides) with the stimuli of average complexity (12 sides) did not yield significant differences in either group. In both groups, however, there was by a $t$ test a statistically significant $(p<.025)$ dominance of the 12-sided and the 24-sided figures over the 4-sided figures.

Novelty. The ordering of means in Table 1 suggests a tendency for the NS and DS groups to attend more to the novel stimuli. The introduction of a set to look at the preferred stimulus reversed this FD. An analysis of variance showed no significant differences between the NS and DS groups with respect to the FD of novel stimull. When their results are combined, the FD of novel stimuli is statistically significant, $t=2.818, d f=31, p<.01$. The $S s$ in the $D S$ and NS groups were each shown four stimulus slides comparing novel with non-novel line drawings. When the Ss in these two groups are pooled the resulting mean FD of the novel figure in each of the four slides is greater than .50. Although it was predicted that the PS group would demonstrate greater FD for more novel stimuli, all four stimulus slides shown this group resulted in FD of the non-novel drawing. This FD, however, was not significant.

\section{Correlations Befween FD and Judged FD}

Phl coefficients were obtained between the Ss' reported FD and their actual FD for each of the 64 slides. The correlations ranged from .72 to 1.00 for 
the PS group, .29 to .97 for the DS group, and .26 to .93 for the NS group. Mean correlations over all slides for the groups are: $P S=.813, D S=.630, N S=$ .598 .

\section{DISCUSSION}

It is apparent that the stimulus properties of both affective tone and structure were effective in influencing the direction of eye movements. Of the 60 mean FD scores obtained from the three groups of Ss, 56 of them were in the direction predicted by the hypotheses. None of the four means not in the predicted direction were significantly different by $t$ test from the chance level of .50 .

The dominance of both the 24-sided and 12-sided figures over the 4-sided figures, and the lack of differential FD for the former two levels of complexity, suggests that the influence of that variable reaches an asymptote at only moderate levels of complexity.

In addition to the effects of stimulus variables, the results point up the importance of instructional sets in research of this kind. A plausible explanation of the role of instructional set is that it influences the weighting of affective tone and information value in determining FD. Thus, the PS group's instructions (to look at the preferred stimulus) may have resulted in a substantial increase in the influence of affective characteristics of the stimuli in determining FD. This unequal weighting would account for the greater FD of more pleasant stimuli in the PS group than in the other two groups and relatively weak and statistically insignificant FD for more complex stimuli by that group. In contrast, instructions for the other two groups may have resulted in a more equal weighting of the two stimulus variables. The less extreme weighting of affective properties thus lead to a less extreme FD of more pleasant stimuli.

Although the intention of this experiment was to investigate the separate effects of affective tone and information value on fixation-dominance, certain characteristics of the stimuli used and the results obtained in the study provide suggestions about the possible interactions of these two variables. It was evident that in some sets of stimuli the negative pictures were considerably more novel and complex than their positive and neutral counterparts. This is most obvious in the set of colored pictures of faces. The novelty of some of the negative pictures, however, did not result in a significant FD of those pictures over less-novel pleasant or neutral pictures. This result suggests that under most conditions affective tone is a more potent determinant of FD than novelty or complexity.

The experiment obviously represents only a first step in the Investigation of the effects of stimulus variables on fixation-dominance. Subsequent research should investigate FD using stimuli that represent numerous points along the continuums of affective tone and information content rather than only two or three such points. Following the investigation of stimull from the two separate continuums, there should be investigations of the joint effects of the two variables on FD. This could be accomplished using stimuli representing systematic combinations of levels of affective tone and complexity or novelty. Alsuther important extension of the present study will be to incorporate developmental levels of the $\mathrm{Ss}$ as an independent variable. It may be that the relative influences of affective tone and information value is a function of age. The results of such research not only would be important in their own right, but, also, they potentially would establish a methodology for the wide employment of eye movements in studies of learning and motivation.

\section{References}

Attneave, F. Physical determinants of the judged complexity of shapes. J. exp. Psychol., 1957, 53, 221-227.

Berlyne, D. E. The influence of complexity and novelty in visual figures on orienting responses. J. exp. Psychol., 1958, 55, 289296.

Cantor, G. N. Responses of infants and children to complex and novel stimulation. In L. P. Lipsitt \& C. C. Spiker (Eds.), $A d-$ vances in child development and behavior. Vol. I. New York: Academic Press, 1963.

Fantz, R. L. Visual perception from birth as shown by pattern selectivity, Ann. N. Y. Acad. Sci, 1965, 118, 793-814.

Luborsky, L.. Blinder, B., \& Mackworth, N. Eye fixation and recall of pictures as a function of GSR responsivity. Percept. mot. Skills, 1963, 16, 469-483.

Mackintosh, N. J. Selective attention in animal discrimination learning. Psychol. Bull., 1965, 64, 124-150.

Nunnally, J. C., Knott, P. D., \& Duchnowski, A. J. Association of neutral objects with rewards: Effects of different numbers of conditioning trials and of anticipated reward versus actual reward. J. exp. child Psychol., in press.

Nunnally, J. C., Stevens, D. A., \& Hall, G. F. Association of neutral objects with rewards: Effect on verbal evaluation and eye-movements. J. exp. child Psychol., 1965, 2, 44-57.

Solley, C. M., \& Santoes, J. Unpublished study report. In C. M. Solley \& G. Murphy (Eds.), Development of the perceptual world. New York: Basic Books, 1960.

Thomas, $\mathbf{H}$. Visual fixation responses of infants to stimuli of varying complexity. Child Develpm., 1965, 36, 629-638.

Thomdike, E. L., \& Lorge, I, The teacher's word book of 30,000 words. New York: Columbia University Press, 1963.

VanderpIas, J. M., \& Garvin, E. A. The association value of random shapes. J. exp. Psychol.. 1959, 57, 147-154.

Webb. W. W., Matheny, A. P., \& Larson, G. Eye movements as a paradigm of approach and avoidance. Percept. mot. Skills, 1963, $16,341-347$.

\section{Note}

1. While this research was in progress the first author was receiving support from a National Defense Graduate Fellowship. The study was supported, in part, by a research grant from the Vocational Rehabilitation Administration, RD-1479-S.

(Accepted for publication March 6, 1967.) 